\title{
Examination and It's Evaluation of Preprocessing Method for Individual Identification in EEG
}

\author{
Masato Yamashita $^{1, a)}$ Minoru Nakazawa $^{1} \quad$ Yukinobu Nishikawa $^{1} \quad$ Noriyuki Abe $^{1}$ \\ Received: June 3, 2019, Accepted: November 7, 2019
}

\begin{abstract}
Recently, the technology of BMI that communicates with humans and operates a robot using human brain information has been actively studied. The authentification function using BMI has been studied by previous research. Although many studies focus on feature extraction and learning model creation, there are few studies that discuss the effectiveness of preprocessing. In this study, we implemented an EEG biometric function using image stimulation method. In this paper, we proposed biometric authentication system system using EEG at time of image stimulus. At the same time, we evaluated the change in authentication accuracy in order to verify the preprocessing (digital filter, artifact countermeasure, epoch) method in the authentication system. As a result, authentication accuracy is improved by performing the proposed preprocessing. In addition, it was shown that convenience and security were improved when using the system.
\end{abstract}

Keywords: brain machine interface (BMI), brain wave, biometric, artifact, preprocessing

\section{Introduction}

In recent years, the technology of Brain Machine Interface (BMI) which conducts communication, robot operation etc. using human brain activity is widely spread. The background is that non-invasive electroencephalograph devices capable of measuring electroencephalogram (EEG) raw data in real time without embedding electrodes in the brain have been released as consumer products. Researches on BMI have been studying to support disabled and intractable patients using autonomously moving wheelchairs [1].

However, there are few products that consider authentication when using their BMI.

Among the widely used authentication methods, the authentication system using ID and password in the mainstream. As another authentication method, biometric authentication system using biometric information (fingerprint, face, etc.), has been also increasing in recent years. Authentication using biometric information has the advantage that spoofing is more difficult than conventional authentication methods using ID and password. However, biometric information such as fingerprints and faces is information that is always exposed to the outside. Therefore, it can be stolen by a camera that records such information. This can lead to serious counterfeit attacks against biometric authentication systems, and the publicly available biometric information can't be changed at its own discretion.

Biometric authentication using EEG has been proposed as a method to cope with these problems. The EEG is internal information of the body and the possibility of being stolen is extremely low because they can't be measured without wearing exclusive

\footnotetext{
Kanazawa Institute of Technology, Nonoichi, Ishikawa 921-8501, Japan
} b1415966@planet.kanazawa-it.ac.jp device. It is also known that EEG changes with external stimulus. Because of this, it is possible to change the registered data after it is stolen.

Considering that BMI is used by intractable patients, authentication and operation can be performed by one device, when incorporating an EEG-based authentication system into BMI. Therefore, it is not necessary for intractable patients to switch devices, and we think that this approach can reduce the burden of users. In the research on biometrics using EEG, there are already some research cases as shown in Chapter 2 [2], [3], [4], [5], [6], [7], [8].

However, many of these studies aim to improve authentication accuracy by feature extraction and improvement of learning models, and there are few cases which highlight the preprocessing of measured EEG. In addition, since the measurement environment, measurement equipment, testers, etc. differ from research to another, it is impossible to compare the authentication accuracy in general.

In this research, we aim to build an authentication system that is used when using a BMI system and can't be stolen from the information. And also, we implement the biometric authentication system using EEG at the time of image stimulus presentation. In this paper, we aim to improve the authentication accuracy by constructing the proposed system and verifying the preprocessing (digital filter, artifact countermeasure, epoch) method.

\section{Related Research}

Performance index of biometric authentication is evaluated by Equal Error Rate (EER). EER is the point where the false rejec-

\footnotetext{
The preliminary version of this paper was published at Multimedia Communication and Distributed Processing System Workshop (DPSWS 2018), November 2018. The paper was recommended to be submitted to Journal of Information Processing (JIP) by the chief examiner of SIGDPS.
} 


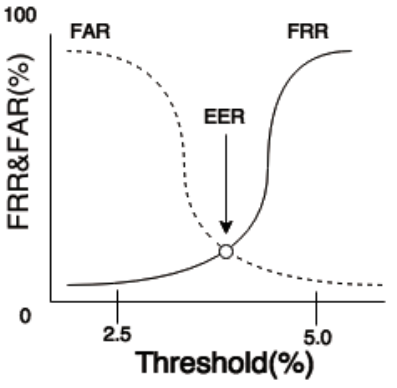

Fig. 1 Curve example of FAR and FRR.

tion rate (FRR) and the false acceptance rate (FAR) are equal. An example of FRR and FAR curves is shown in Fig. 1.

The horizontal axis in Fig. 1 shows is the threshold for judging registrants and intruders, and the vertical axis shows the probability of FAR and FRR. FRR is defined as the rate of false recognition by threshold divided by the total number of matches. In this case, a registrant is recognized as a different person. In addition, FAR is defined as the rate of false recognition at which another person (intruder or another registrant) is incorrectly recognized as a registrant by the threshold value. When the FRR is high and the FAR is low, it is an authentication that emphasizes on security, and when the FRR is low and the FAR is high, it is an authentication that emphasizes on convenience [9]. Therefore, it is required to provide an authentification system which shows low EER, as well as having high levels of both convenience and security.

In biometric authentication using EEG, there are already some research cases. However, as mentioned above, since the condition of measurement environment, measurement equipment, testers, etc. are different for each research, it is impossible to compare the authentication accuracy in general.

Ishikawa et al.'s research classifies registrants without considering intruders. At that time, EER: $3.8 \%$ was obtained by combining five tasks with the power spectrum of the four frequency bands of $\theta$ wave, $\alpha$ wave, $\beta$ wave, and $\gamma$ wave as feature amounts [2].

In research by Ishikawa et al., they aim to EEG authentication in a short time, and they are doing research on biometric authentication using brain waves at rest which not require mental tasks or external stimulus. By using AdaBoost for the feature values obtained from plural feature, a learning model was created, and EER: $0.52 \%$ was obtained as having 32 registrants and 18 intruders [3].

As research for biometric authentication system using video stimuli, Yoshikawa and colleagues divided the $\alpha$ wave band and the $\beta$ wave band of 30 testers who are driving a car into a plurality of regions and studied only the frequency band with good authentication rate by using machine learning. They obtained EER: $31 \%$ [4].

In the study of Touyama et al., they used auditory stimuli, deriving personal identification accuracy using machine learning method under three conditions, such as indoor resting seating condition, outdoor resting upright condition, outdoor walking condition. From their research, they mentioned the possibility of brain wave personal authentication. About seven testers realized an individual authentication accuracy rate of about $87 \%$ at indoor

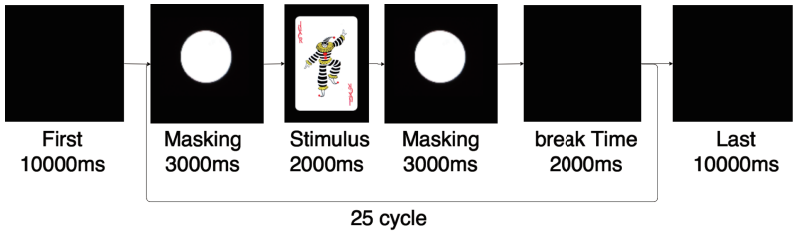

Fig. 2 Flow of one trial.

resting seating [5].

In the research by Yazani et al., the k-near method was used using the peak of the power spectral density (PSD) of the gamma band VEP signal (GMVEP) as a feature value. They classified 20 registrants and got $100 \%$ classification accuracy when $K=14$ or more [6]. In the study of Yeom et al., self-face and non-selfface images were used as stimuli. ERP (Event related potential) was used as features when the registrant saw self-face and nonself-face. Then, $86.1 \%$ recognition accuracy was obtained for 10 registrants [7]. In their research, it is necessary to look at the face of all registrants exhaustively, and there is a problem that the time taken for certification increases as the number of registrants increases. In the study of Palaniappan et al., Gamma band spectral power (GBSP) during visual stimulation was used as a feature quantity, and accuracy was improved by performing principal component analysis (PCA) and normalization as preprocessing. Using three classifiers as learning models and using PCA recognition accuracy and up to $96.50 \%$ recognition accuracy [8].

\section{Measurement Method of EEG}

In this section, we describe a method for acquiring EEG using image stimulation.

We use Emotiv Epoc+ as a device to acquire Electroencephalograph. Emotiv Epoc+ is a non-invasive device and can measure brain activity at a sampling frequency of $128 \mathrm{~Hz}$ using 14 electrodes. 14 electrodes are mounted to a tester's head according to International 10-20 method [10]. OpenVIBE is used to obtain EEG and to present image stimuli. OpenVIBE is a software platform that can measure EEG while presenting image stimuli in real time.

Figure 2 shows the flow of the image displayed on the monitor when measuring the EEG.

In our research, five cards (Heart A, Clover A, Heart J, Clover J, Joker) are used as image stimuli. In the EEG measurement, an image with only black background is displayed to measure the resting EEG during the first and last 10 seconds. After that, in order to focus on the eyes, the white circle is displayed for 3 seconds, the playing card is displayed for 2 seconds, the white circle is displayed for 3 seconds, and the black background is displayed for 2 seconds. Repeat the flow 25 times, from the display of the white circle to the display of the black background for 2 seconds, in order to focus on the gaze. The order of presenting the images is random, and each kind of image must be displayed five times. The flow shown in Fig. 2 is one trial, and the time required for one trial measurement is about 270 seconds. As an environment for measurement, the tester was in the indoor sitting position, and the experiment was performed with the distance between the tester and the monitor fixed at $80 \mathrm{~cm}$. Assuming use 
in a real environment, measurements were made in a room where living environment sounds, without using a special environment to prevent noise such as a shielded room.

\section{Proposed Method}

In this section, we describe a biometric authentication system based on EEG analysis. We show the outline of the biometric authentication system based on EEG analysis (Fig. 3). The system is divided into a registration phase and an authentication phase.

In the registration phase, a registration number is assigned to each registrant, and their EEG are measured.

As the EEG are measured using non-invasive devices, the measurement contains a lot of noise as described later. Therefore, preprocessing for noise removal is performed before feature extraction.

Feature extraction uses the similarity of time series data between the electrodes of the section looking at the image stimulus as the feature by the cross correlation coefficient $(C C)$. By this feature extraction, the feature for each image stimulus is used to create a learning model. In the authentication phase, authentication is performed without knowing the certifier's registration number. The same preprocessing and feature extraction as performed in the registration phase are performed on the EEG data. Classification and authentication are performed using the learning model created in the registration phase. In the following sections, preprocessing, feature extraction, creation of a learning model and classification/authentication method will be described in detail.

\subsection{Preprocessing}

From the point of using a non-invasive devices and measuring indoor where the living environment sound occurs, it is conceivable that the measured electroencephalogram data contains many artifacts. Therefore, artifacts must be removed appropriately. As we call, an artifact is all noise other than EEG. Because the EEG is very weak, electrical potentials other than EEG are likely to be mixed in the measurement [11]. It is necessary to remove artifacts contained in the raw data by performing preprocessing on the measured EEG data.

Preprocessing performs the following three methods.

(1) Digital filter

(2) Artifact countermeasure

(3) Epoch

Each method will be specifically described in the following section.

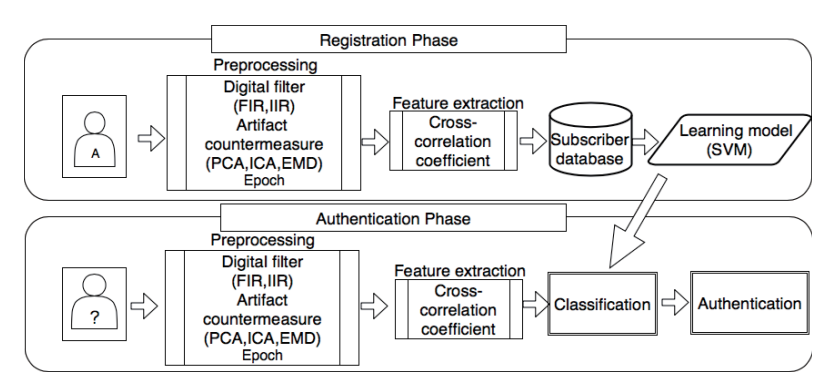

Fig. 3 Authentication system overview.

\subsubsection{Digital Filter}

The digital filter lets pass only specific frequency components and reduces other frequency components. There are roughly two types of digital filters: FIR (Finite impulse response) filter and IIR (Infinite impulse response) filter [12]. Impulse is a meaning of the waveform when a filter is applied to an instantaneous voltage change. The characteristic of FIR is the meaning of the influence of the voltage change at a certain moment only spreads to a limited time point. IIR has the effect of voltage change at a certain moment remains forever. In this study, we use least squares approximation bandpass filter for FIR filter and Butterworth bandpass filter for IIR filter. By using the band pass filter, frequency bands other than a specific frequency band are reduced. The frequency band allowed to pass by the digital filter is $4-40 \mathrm{~Hz}$. This frequency band is a frequency band where activities of EEG are mainly observed.

\subsubsection{Artifact Countermeasure}

Artifact countermeasures are performed using principal component analysis (PCA), independent component analysis (ICA), and empirical mode decomposition (EMD). PCA is one of the methods for unsupervised learning, and this obtains a linear transformation in a direction in which the variance of learning data is maximized [13]. The feature value linearly transformed with the eigenvector corresponding to the largest eigenvalue is called the first principal component $\left(\lambda_{1}\right)$, and then the feature transformed by the eigenvector corresponding to the $k$-th eigenvalue is the $k$-th principal component $\left(\lambda_{k}\right)$. How much information the main component has is called the contribution ratio of the main component. When the total number of principal components is $n$, the cumulative contribution rate $\left(r_{k}\right)$ up to the $k$-th component is expressed by Eq. (1).

$$
r_{k}=\frac{\sum_{i=1}^{k} \lambda_{i}}{\sum_{i=1}^{n} \lambda_{i}}
$$

We use singular value decomposition as an algorithm of principal component analysis. Singular value decomposition is one of matrix decomposition methods for matrices with complex numbers or real numbers as components. This method presents less disadvantages than finding the covariance matrix.

ICA is a method for estimating the original signal of an independent component (IC) separated from the signal observed by a plurality of sensors. An example of the relationship between the original signal and the observation signal is shown in Fig. 4 (partly modified in the figure from Ref. [14]).

In Fig. 4 , the observation signal is $X_{i}(t)$ and the original signal is $S_{j}(t)$. In the observation signal, multiple original signals

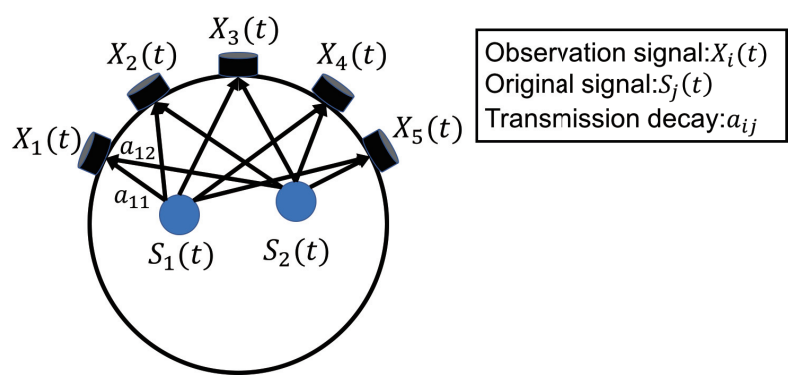

Fig. 4 Relationship between original signal and observation signal. 
are attenuated by transmission decay $a_{i j}$ and observed in a mixed state [14]. FastICA is applied to the ICA algorithm [15]. We used maximization and minimization of kurtosis as the evaluation function, and used the fixed point algorithm as the optimization.

EMD is a time-frequency analysis method for unsteady signals [16]. And it divides each frequency mixed in the measured signal. By using EMD, the signal $x(t)$ is separated into a plurality of signals called Intrinsic Mode Function (IMF). Assuming that $x(t)$ is an input signal, the total number of signals obtained by EMD is $n$, each signal obtained by EMD is $k_{i}(t)$, and the residual is $r(t)$, the following Eq. (2) can be expressed.

$$
x(t)=\sum_{i=1}^{n} k_{i}(t)+r(t)
$$

The IMF resulting from EMD satisfies the following two requirements.

1. The number of IMF extrema (the sum of the maximum value and the minimum value) and the number of zero crossings are equal, at most 1 difference.

2. At any point in the signal, the average value of the envelope (upper and lower envelopes) created from the maximum and minimum points is zero.

We show the procedure for artifact countermeasure (Fig. 5).

The measured EEG data of 14 channels for one trial is $V(t)$ ( $t=0-T \mathrm{~ms}$ ). $T \mathrm{~ms}$ is the time for one trial.

Step1. PCA is applied to $V(t)$, and the number of principal components $(k)$ when the cumulative contribution ratio of the principal components exceeds $80 \%$ is $N_{1}$.

Step2. Apply ICA to $V(t)$ and separate it into $N_{1}$ ICs. $I C_{n_{1}}=$ $\left\{i c_{1}, \cdots, i c_{n_{1}}\right\}$ be $N_{1}$ sets of ICs separated.

Step3. Apply EMD to each component of $I C_{n_{1}}$. Let $N_{2}$ be the number of IMFs obtained from $i c_{i}\left(i=1, \cdots, n_{1}\right)$ and $K_{n_{2}}=\left\{k_{1}, \cdots, k_{n_{2}}\right\}$ be the set of IMFs.
Step4. For each component $K_{j}\left(j=1, \cdots, n_{2}\right)$ of $K_{n_{2}}$, fast Fourier transform is performed and divided into five frequency bands. Frequency bands are $\delta$ wave $(1-3 \mathrm{~Hz}), \theta$ wave (4-8 Hz), $\alpha$ wave $(9-13 \mathrm{~Hz}), \beta$ wave $(14-23 \mathrm{~Hz}), \gamma$ wave $(24 \mathrm{~Hz}-)$. The frequency band in which EEG activity is actively observed is mainly $4-40 \mathrm{~Hz}$. Therefore, $K_{n_{2}}=$ $\left\{k_{1}, \cdots, k_{n_{2}}\right\}$ which contains $\delta$ wave the most in a divided frequency band is eliminated as noise.

Step5. We reconstruct the set of independent components after removal of noise $\left(I C_{n 1}^{\prime}=\left\{i c_{1}^{\prime}, \cdots, i c_{n_{1}}^{\prime}\right\}\right)$ using the remaining set of $K_{n_{2}}$.

Step6. Reconstruct as $V^{\prime}(t)$ using set $I C_{n_{1}}^{\prime}$.

4.1.3 Epoch

The epoch is to cut out image stimulation intervals for each time window. In this research, it is used to cut out the section in which the image stimulus is presented from the designated part for the specified number of seconds.

\subsection{Feature Extraction}

The feature value is extracted from the data after preprocessing. The cross-correlation coefficient $(C C)$ between the two electrodes $(a, b)$ is expressed by Eq. (3) [17].

$$
C C=\frac{\sum_{k=0}^{N-1} a_{k} b_{k}}{\sqrt{\sum_{k=0}^{N-1} a_{k}^{2}} \sqrt{\sum_{k=0}^{N-1} b_{k}^{2}}}
$$

$N$ is the data length at the time of extraction by epoch. $a_{k}$, $b_{k}$ are time series data of electrodes. The cross-correlation coefficient $(C C)$ given by Eq. (3) has a value from -1 to +1 , and it becomes a normalized correlation coefficient. The combination of all the electrodes is the feature quantity. Therefore, 91 feature quantities are obtained. The reason why the cross-correlation coefficient is used is that it is considered that the location and time at which the EEG is activated when the same stimulus is presented

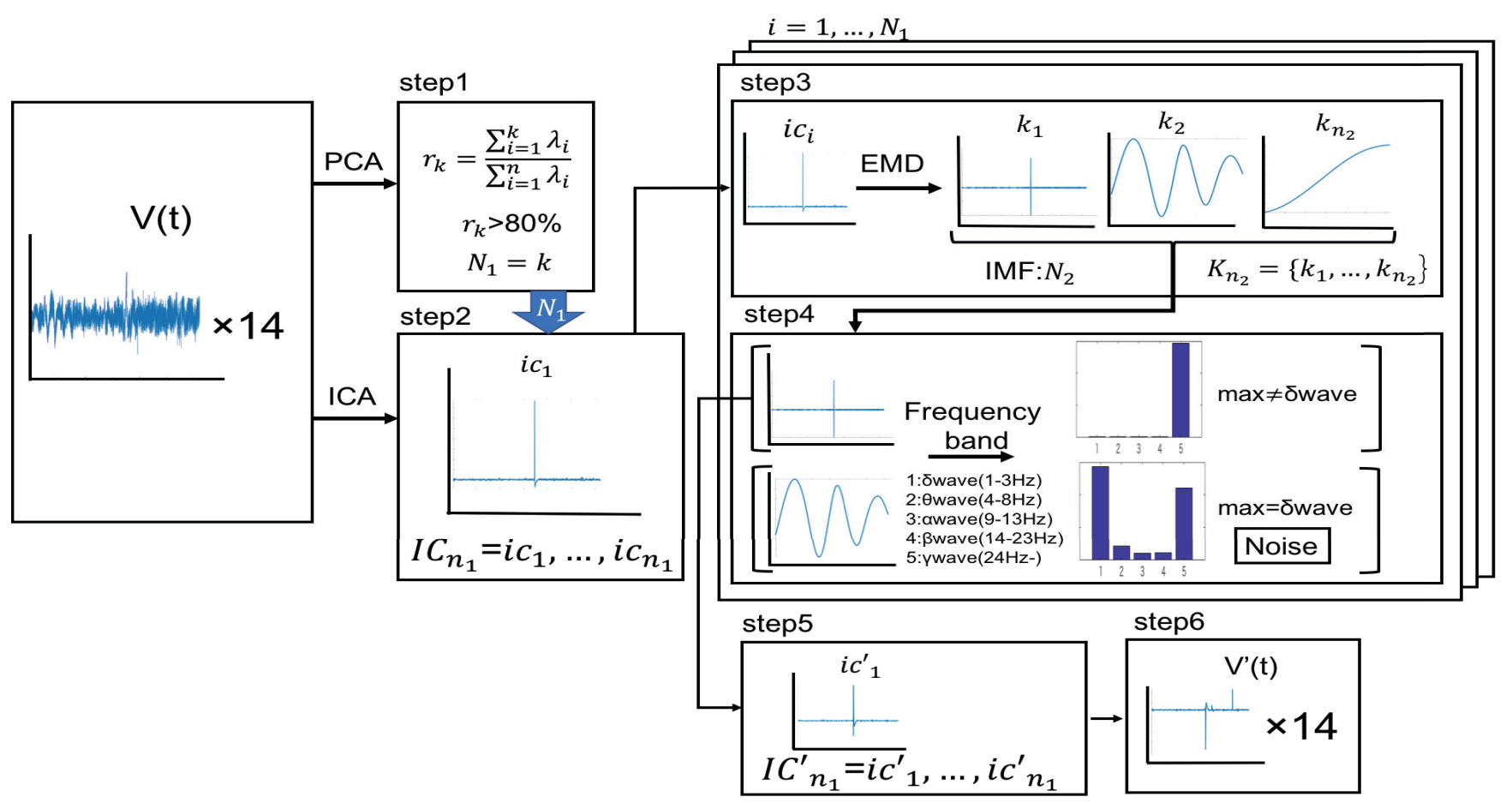

Fig. 5 Flow of artifact countermeasure. 
may vary depending on the person.

\subsection{Learning Model Creation/Authentication Method}

We applied Support Vector Machine (SVM) as a method for creating a learning model, classifying and authenticating from feature quantities obtained by feature extraction. SVM is one of pattern recognition learning models [18]. It is often used as a method to classify two classes basically. However, since we need to classify multiple registrants in this research, we use it for multi-class classification. There are two types of multi-class classification in SVM: one-to-multi and one-to-one method. The one-to-multi method has the advantage that the number of calculations can be reduced since the two-class classifier is trained by the number of classes (number of registrants). However, when the total count of one class is very high relative to the count of the other class, the results will be biased towards the other classes.

In the one-to-one method, it is necessary to create $((C-1) C) / 2$ classifiers, as the number of registrants is $C$, to create classifiers for all class pairs. Although the number of classifiers increases compared to the one-to-multi method, it has the advantage of reducing the computational cost for one classifier. In this research, we perform multi-class classification using one-to-one method. In the SVM, it is necessary to calculate the inner product of the feature vectors, but by using the kernel function, it is possible to create a model using curves without calculating inner product. As a kernel function, we use a radial basis function (RBF: Radial Basis Function). The RBF kernel $(G)$ is given by the following equation.

$$
G\left(x_{j}, x_{k}\right)=\exp \left(-\left\|x_{j}-x_{k}\right\|^{2}\right)
$$

When using a learning model, the scaling process is performed on the feature value to make the value range from 0 to +1 . This is to prevent loss of information at the time of calculation.

During SVM learning, parameters are not adjusted using hyperparameters. This is to eliminate the effect of accuracy due to SVM parameter adjustment when verifying the effectiveness of preprocessing.

In the registration phase, a learning model is created for each type of playing card. In the authentication phase, the confidence ratio is obtained for each registrant in the learning model by passing the feature value of the certifier. Calculation of the confidence ratio is done using Kullback-Leibler divergence [19]. When the maximum confidence ratio obtained in the authentication phase is lower than the preset threshold, the authentication is refused as an intruder. And when it is higher than the threshold, it is authenticated as the registrant who gets the maximum confidence ratio.

\section{Validation Method and Evaluation}

In this section, we show the verification method of this proposed system and the evaluation result.

Authentication accuracy is determined by the EER described in Chapter 2. Because of that, it is necessary to obtain FAR and FRR. Based on the confidence ratio of each registrant obtained in the authentication phase, FAR obtains the rate of accepting a certifier who is not a correct registrant as a registrant based on the threshold value. Based on the reliability of each registrant obtained in the authentication phase, FRR obtains the rate at which the authentication fails for each threshold even though the authenticator is a correct registrant.

To measure EEG, 31 male university students cooperated as testers. When measuring the testers EEG, the flow of measurement was explained once before the measurement, and consent was obtained for participation. We also explained the display image and the points measurement. Testers took a break for a fixed time after one trial. The electroencephalograph was still mounted during the break. One trial was measured three times in one day for one tester. 31 students will be verified as 20 registrants and 11 intruders. There were 15 data for one image stimulus of playing cards. The validation method is validated using 15 cross validations. FAR and FRR obtained from 15 were averaged to obtain an average EER. In the following discussion, the EER value is assumed to be average.

\subsection{Verification Condition}

Verification is performed by combining validation conditions of preprocessing (Digital filter, Artifact countermeasure, Epoch). Validation conditions are used to verify the combination at the lowest EER. The conditions of each preprocessing are shown in the Table 1.

The number of dimensions of the digital filter is fixed by 4dimensional, band pass filter, and the frequency band to pass is fixed at $4-40 \mathrm{~Hz}$ by band pass filter. By the epoch, the length of the image stimulation interval taken out is $1,000 \mathrm{~ms}$ or $2,000 \mathrm{~ms}$. The sampling frequency of the electroencephalograph is $128 \mathrm{~Hz}$, so there are 256 data points in $2,000 \mathrm{~ms}$. At the time of $1,000 \mathrm{~ms}$, there are 129 positions, starting from 1 to 129 in which data points are cut out. Therefore, there are 130 validation patterns of epochs.

\subsection{Evaluation Result}

At first, only the EEG data, when one card image was viewed was used for the authentication, and it was verified to understand the combination of the condition for the preprocessing with which the EER was most reduced.

Fixing the condition number $\mathrm{C} 2$ for epoch, and checking the condition number B1-B2 for artifact countermeasure and the condition number A1-A5 for digital filters, the results of verification are shown in Fig. 6.

On Fig. 6, the vertical axis is EER (\%), and the number of the horizontal axis is the condition number of the digital filter. As a

Table 1 Condition of each preprocessing.

\begin{tabular}{l|c}
\hline \hline Condition number (A) & digital filter \\
\hline A1 & No digital filter \\
\hline A2 & IIR filter for one whole trial \\
\hline A3 & FIR filter for one whole trial \\
\hline A4 & IIR filter for data after epoch \\
\hline A5 & FIR filter for data after epoch \\
\hline Condition number (B) & Artifact countermeasure \\
\hline B1 & No artifact countermeasure \\
\hline B2 & With artifact countermeasure \\
\hline Condition number (C) & $\begin{array}{c}\text { Epoch: Length to cut out } \\
\text { (Start point of data point to cut out) }\end{array}$ \\
\hline C1 & 1,000 ms (Starting point: 1 to 129) \\
\hline C2 & 2,000 ms(1) \\
\hline
\end{tabular}




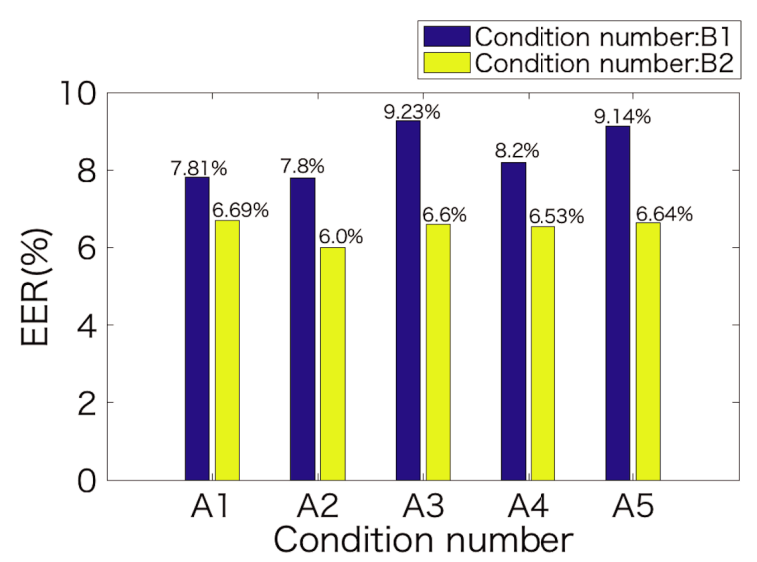

Fig. 6 Authentication result 1.

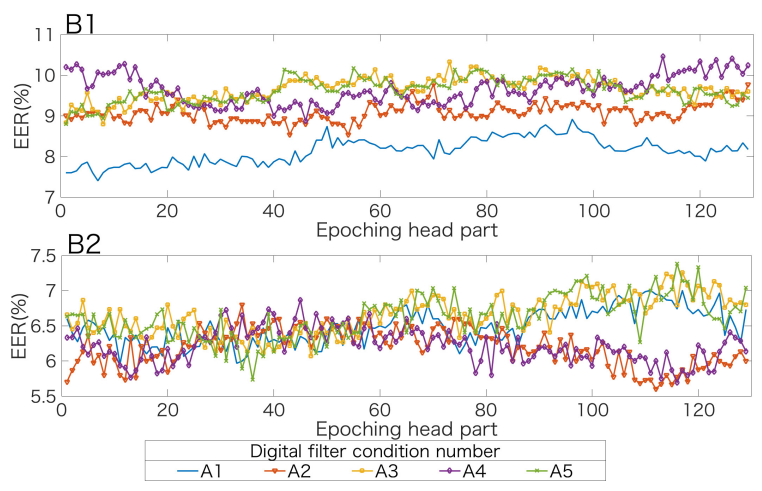

Fig. 7 Authentication result 2.

Table 2 Minimum EER under each condition in Fig. 7.

\begin{tabular}{l|l|l|l}
\hline \hline Condition number & EER (\%) & Condition number & EER (\%) \\
\hline A1-B1-C1 (7) & 7.400 & A1-B2-C1 (11) & 5.957 \\
\hline A2-B1-C1 (43) & 8.535 & A2-B2-C1 (112) & 5.602 \\
\hline A3-B1-C1 (8) & 8.797 & A3-B2-C1 (47) & 6.068 \\
\hline A4-B1-C1 (46) & 8.871 & A4-B2-C1 (116) & 5.693 \\
\hline A5-B1-C1 (1) & 8.795 & A5-B2-C1 (36) & 5.733 \\
\hline
\end{tabular}

result, artifact countermeasure was applied to the measured EEG, and it was confirmed that EER was lower than the case without EER.

The result of fixing the condition number $\mathrm{C} 1$ of Epoch, 1-129 the Starting point of the Epoch, the condition number B1-B2 for artifact countermeasure, and the condition number A1-A5 of the digital filter is shown in Fig. 7. On Fig. 7, the vertical axis is EER (\%), and the horizontal axis is the starting point of the epoch. The graph B1 shows the condition number B1 and the graph B2 shows the condition number B2. Each type of line graph corresponds to the condition number of the digital filter.

Table 2 shows the minimum EER under each condition in the graph shown in Fig. 7. In the condition number, each condition number is entered in the order of digital filter - Artifact countermeasure - Epoch (Starting point).

The minimum EER was obtained at the time of condition A2B2-C1 (Starting point: 112, Starting time: 0.867 s) from the combination of all these conditions. At that time, EER was $5.602 \%$.

Starting time is the time to start cutting. It is expressed as Starting time $[\mathrm{s}]=($ Starting point -1$) /$ Sampling rate . Sampling rate is an electroencephalography sampling rate (128).

Next, we verified how EER changes when authentication was
Table 3 lowest EER at multiple image stimuli.

\begin{tabular}{r|c|l}
\hline \hline Number of image stimulus & EER $(\%)$ & Condition number \\
\hline 1 & 5.602 & $\mathrm{~A} 2-\mathrm{B} 2-\mathrm{C} 1(112: 0.867 \mathrm{~s})$ \\
\hline 2 & 2.419 & $\mathrm{~A} 2-\mathrm{B} 2-\mathrm{C} 1(104: 0.804 \mathrm{~s})$ \\
\hline 3 & 1.748 & $\mathrm{~A} 1-\mathrm{B} 1-\mathrm{C} 1(1: 0 \mathrm{~s})$ \\
\hline 4 & 1.202 & $\mathrm{~A} 1-\mathrm{B} 1-\mathrm{C} 1(2: 0.007 \mathrm{~s})$ \\
\hline 5 & 0.944 & $\mathrm{~A} 1-\mathrm{B} 1-\mathrm{C} 1(34: 0.257 \mathrm{~s})$ \\
\hline
\end{tabular}
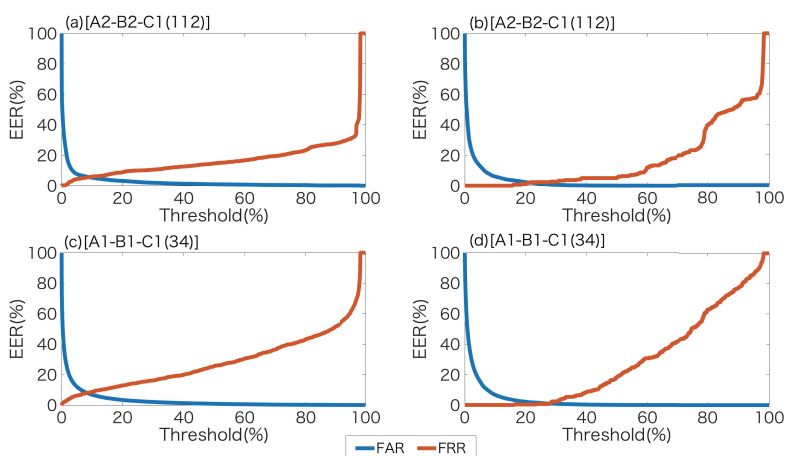

Fig. 8 Graph of FAR and FRR.

performed using multiple card images. When using a plurality of playing card images in the authentication phase, the credibility rates of each registrant obtained from the learning model of each playing card are added together, averaged and classified and authenticated. At that time, the minimum EER obtained is shown in Table 3 for each number of cards and their combination. In the condition number, each condition number is entered in the order of digital filter - Artifact countermeasure - Epoch (Starting point: Starting time [s]).

Table 3 shows that EER decreases by increasing the number of image stimuli. In authentication, it is thought that it is more effective as an authentication method to use electroencephalogram data that saw multiple image stimuli than viewing a single image stimulus. However, when using multiple image stimuli, there is a tradeoff that the time required for authentication increases because the time during which the user is viewing the image stimulation increases. Also, when the number of image stimuli during authentication is 1 and 2, the artifact countermeasure showed the minimum EER. However, when it is more than 3, the result shows the smallest EER without performing digital filter and artifact measures.

The graph of FAR and FRR verified with 1 image and 5 image conditions is shown in Fig. 8.

The graphs (a) and (b) are the condition numbers A2-B2-C1 (112). (a) is when the number of image stimuli used for authentication is one, and (b) is when the number of image stimuli is five. Similarly, the graphs of (c) and (d) are condition numbers A1-B1-C1 (34). (c) is when the number of image stimuli used for authentication is one, and (d) is when the number of image stimuli is five.

Graph (a) had an EER of $5.602 \%$, while graph (c) had an EER of $8.002 \%$.

Looking at the graphs of (a) and (c) shown in Fig. 8, the FAR is almost the same, whereas the FRR of (a) is lower than (c). It can be seen that the FRR rapidly increases near the threshold value of $95 \%$. Graph (b) was EER: $2.000 \%$, while graph (d) was EER: $0.944 \%$. The details of FRR for each threshold in graph (b), (d) 
Table 4 FRR details of Fig. 8 (b), (d).

\begin{tabular}{r|r|r}
\hline \hline & FRR (\%) & FRR (\%) \\
threshold (\%) & (b) [A2-B2-C1 (112)] & (d) [A1-B1-C1 (34)] \\
\hline 10 & 0 & 0 \\
\hline 20 & 1.33 & 0.33 \\
\hline 30 & 2.66 & 2.33 \\
\hline 40 & 5.00 & 8.66 \\
\hline 50 & 5.33 & 18.33 \\
\hline 60 & 12.00 & 30.66 \\
\hline 70 & 19.33 & 41.66 \\
\hline 80 & 39.33 & 63.00 \\
\hline 90 & 52.66 & 77.33 \\
\hline
\end{tabular}

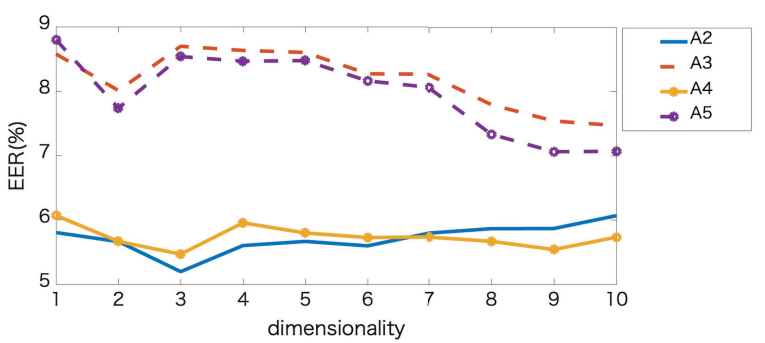

Fig. 9 Accuracy of authentication by the number of dimensions of digital filter.

are shown in Table 4

It can be confirmed that the value of (d) is lower than that of (b) at threshold value: $30 \%$. This is considered that the reliability of the correct registrant has dropped locally due to the digital filter and the countermeasure against the artifact. However, it can be seen from Table 4 that the FRRs of (b) are all lower than the FRRs of (d) when the threshold is $40 \%$ or more. From this, it was suggested that applying artifact countermeasure and digital filters as preprocessing is effective to improve user's convenience.

\subsection{Verification and Result of Authentication Accuracy by Changing the Dimensionality of Digital Filter}

Next, we verified the authentication accuracy when the dimensionality of the digital filter is changed.

Let B2-C1 (112) be the fixed verification condition. By changing the dimensionality in A2 to A5 of the conditions of the digital filter shown in Table 1 to $1-10$ dimensions, it is verified which condition is optimal when performing the digital filter. The frequency band to pass in the digital filter is fixed at $4-40 \mathrm{~Hz}$. The evaluation is EER when the number of image stimuli is one. The verification results are shown in Fig. 9.

According to Fig. 9, changing the dimensionality of the digital filter resulted in the minimum EER (5.19\%) when A2 (one trial IIR filter) was executed in three dimensions.

\subsection{Verification and Result of Accuracy by Combination of Registrants}

We verified the authentication accuracy when the combination a registrant and an intruder is changed. We choose 1,000 of all the patterns of a registrant and an intruder at random, and then tested. Verification conditions were A2-B2-C1 (112). The dimensionality of the digital filter was three-dimensional. A1-B1-C2 in the combination of the same registrant and intruder was compared. The effectiveness of preprocessing is verified using average FRR and average EER, which are averages of 1,000 verification results
Table 5 average FRR, average EER, average reduction rate by combination of registrants.

\begin{tabular}{r|r|r|r}
\hline \hline $\begin{array}{r}\text { threshold } \\
\text { value (\%) }\end{array}$ & $\begin{array}{r}\text { A2-B2-C1 (112) } \\
\text { average FRR (\%) }\end{array}$ & $\begin{array}{r}\text { Average } \\
\text { average FRR (\%) }\end{array}$ & $\begin{array}{r}\text { reduction } \\
\text { rate (\%) }\end{array}$ \\
\hline 10 & 6.00 & 10.90 & 4.90 \\
\hline 20 & 8.18 & 17.00 & 8.82 \\
\hline 30 & 10.31 & 22.27 & 11.96 \\
\hline 40 & 12.13 & 27.63 & 15.49 \\
\hline 50 & 13.91 & 33.65 & 19.73 \\
\hline 60 & 15.57 & 39.65 & 24.08 \\
\hline 70 & 17.57 & 45.78 & 28.22 \\
\hline 80 & 21.02 & 53.35 & 32.33 \\
\hline 90 & 26.53 & 65.10 & 38.58 \\
\hline & average EER (\%) & average EER (\%) \\
\hline & 5.87 & 9.43 & 3.57 \\
\hline
\end{tabular}

for a single image stimulus used for authentication, as evaluation values. The average FRR and average EER for each threshold value in verification and the average reduction rate are shown in Table 5.

From Table 5, it was confirmed that both FRR and EER decreased by preprocessing. The average decreasing rate of EER is $3.57 \%$, but the average decreasing rate is $38.58 \%$ when the threshold is $90 \%$ for FRR. This indicates that the convenience and security of the user is improved compared to the time when the preprocessing is not applied. Moreover, the combination of registrants was changed, and when the condition of pretreatment was A2-B2-C1 (112), it showed average EER: 5.87\%, minimum EER: $5.00 \%$ maximum EER: $6.96 \%$ standard deviation: 0.275 , Dispersion: 0.076. From this, we think that the influence on the change of the value of EER by registrant is small.

\section{Conclusion}

In this paper, we examined and evaluated the preprocessing method for personal identification in EEG. Among the proposed methods, combinations of preprocessing, authentication using multiple image stimuli, the number of dimensions of digital filters, and changes in authentication accuracy for combinations of registrants were verified. As a result, the minimum EER was obtained when the data after $0.867 \mathrm{~s}$ (Starting point: 112) from the image stimulus presentation was cut out for $1,000 \mathrm{~ms}$ by artifact countermeasure, digital filter (Three-dimensional IIR filter for a single trial) and epoch as preprocessing when performing authentication. We examined the influence of authentication accuracy by the combination of a registrant and an intruder, and it is considered that the influence on the change of EER value by registrant is small. In addition, the average decreasing rate of EER by preprocessing was $3.57 \%$, and the average decreasing rate of FRR was $38.58 \%$ when the threshold was $90 \%$. These results suggest that preprocessing improves the accuracy of authentication and improves the convenience of the user. As future work, this paper uses EEG data of one tester without separating the data for the registration and authentication, and the tester didn't take off the electroencephalograph in a experiment day. Therefore, it is considered to be necessary to separate measurement for the authentication and the registration for authentication, and to verify whether authentication of EEG is possible even after one week. Next, in this research, the tester's condition was indoor rest sitting 
position authentication. It is necessary to create a learning model in sitting position state at the time of registration, and to verify whether authentication can be performed even if EEG measured in a state other than sitting position state in the authentication phase. Furthermore, although the image of playing cards was used for image stimulation this time, we will investigate what kind of changes occur in the reactions of the EEG by using other image stimuli. It is also necessary to verify if any individual differences can be made depending on the types of image stimulus.

\section{References}

[1] Nakazawa, M., Takanohashi, K. and Abe, T.: Implementation and it's Evaluation of Autonomous Moving WheelChair System using EEG, IPSJ SIG Technical Report, Vol.2015-MBL-75, No.14, pp.1-8 (2015).

[2] Ishikawa, Y., Nishibata, K., Takata, M. and Joe, K.: Feature Extraction for Electroencephalographic Personal Identification, IPSJ SIG Technical Report, Vol.2014, No.20, pp.1-6 (2014).

[3] Ishikawa, Y., Nishibata, K., Takata, M. and Joe, K.: Biometric Authentication Based on Multi-feature Combination Using EEG, IPSJ Trans. Mathematical Modeling and Its Applications, Vol.10, No.1, pp.23-32 (2017).

[4] Yoshikawa, T., Fukuda, H., Nakanishi, I. and Li, S.: Person Authentication using EEG - Verification Based on 1vs1 SVM using Divided EEG Spectra (2013).

[5] Touyama, H.: EEG-Based Biometry in Outdoor Environment toward the Operation of a Portable BMI System - A Study with Small Number of People during Standing or Ambulatory Condition, Journal of Japan Society for Fuzzy Theory and Intelligent Informatics, Vol.26, No.2, pp.606-616 (2014).

[6] Yazdani, A., Roodaki, A., Rezatofighi, S.H., Misaghian, K. and Setarehdan, S.K.: Fisher linear discriminant based person identification using visual evoked potentials, 2008 9th International Conference on Signal Processing, pp.1677-1680 (online), DOI: 10.1109/ICOSP.2008.4697459 (2008).

[7] Yeom, S.-K., Suk, H.-I. and Lee, S.-W.: Person authentication from neural activity of face-specific visual self-representation, Pattern Recognition, Vol.46, No.4, pp.1159-1169 (online), DOI: https://doi.org/10.1016/j.patcog.2012.10.023 (2013).

[8] Palaniappan, R. and Ravi, K.V.R.: Improving Visual Evoked Potential Feature Classification for Person Recognition Using PCA and Normalization, Pattern Recogn. Lett., Vol.27, No.7, pp.726-733 (online), DOI: 10.1016/j.patrec.2005.10.020 (2006).

[9] Information-technology Promotion Agency, Japan: Seitaininnsyoudounyuu - unnyounotamenogaidorainn [Guidelines for introduction and operation of biometrics] (2009).

[10] Jasper, H.H.: The ten twenty electrode system of the international federation, Electroencephalography and Clinical Neurophysiology, Vol.10, pp.371-375 (1958).

[11] Ushiro, K.: A-tifakutotaisaku [Artifact countermeasure], Japanese Journal of Clinical Neurophysiology, Vol.42, No.6, pp.393-398 (2014).

[12] Nittono, H. and Onoda, K.: Effects of filtering on event-related potential waveforms, Japanese Journal of Physiological Psychology and Psychophysiology, Vol.26, No.3, pp.237-246 (2008).

[13] Jolliffe: Principal Component Analysis, Springer (2002).

[14] Murata, N.: nyuumondokurituseibunnbunnseki [Introductory independent component analysis], Tokyo Denki University Press (2004).

[15] Hyvärinen, A. and Oja, E.: Independent component analysis: Algorithms and applications, Neural Networks, Vol.13, pp.411-430 (2000).

[16] Huang, N.E.: The empirical mode decomposition and the Hilbert spectrum for nonlinear and non-stationary time series analysis, Proc. Royal Society of London, A, Vol.454, pp.903-995 (1998).

[17] Hedges, L.V. and Olkin, I.: Statistical Methods for Meta-Analysis, Academic Press (1985).

[18] Vapnik., V.: Statistical Learning Theory, Wiley (1998).

[19] Dietterich, T. and Bakiri, G.: Solving Multiclass Learning Problems Via Error-Correcting Output Codes, Journal of Artificial Intelligence Research, Vol.2, pp.263-286 (1995).

\section{Editor's Recommendation}

This paper proposes and evaluates a biometric identification system using EGG with the image stimulus presentation. The system with BMI is difficult to eavesdrop and achieves $98 \%$ ac- curacy of the personal identification. The paper gives insights to readers in this research field and thus is selected as a recommended paper. (Chief examiner of SIGDPS Atsushi Tagami)

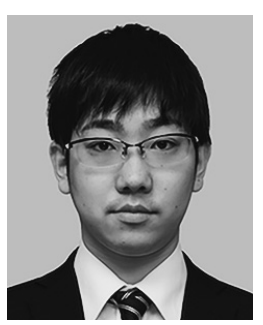

Masato Yamashita received his B.E. degree from Kanazawa Institute of Technology in 2018. He is currently M.E. student at Graduate School of Engineering, Kanazawa Institute of Technology. His research interest include Brain Machine Interface and Artificial Intelligence. $\mathrm{He}$ is a student member of the IPSJ.

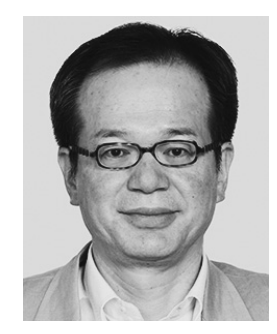

Minoru Nakazawa received his M.E., and Ph.D. degrees from Kanazawa Institute of Technology, Ishikawa, Japan, in 1993, 1999, respectively. In 1993, he joined Fujitsu Laboratory. He became an assistant, professor at Kanazawa Institute of Technology, in 1996, 2011. His research interests include Autonomous Distributed System, Robotics and Artificial Intelligence, $\mathrm{He}$ is a member of IPSJ, IEICE, and IEEE.

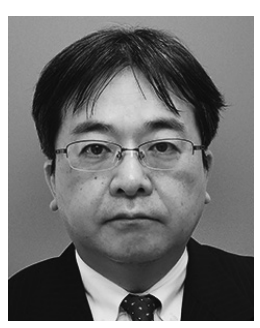

Yukinobu Nishikawa received his B.E. degree from Osaka University, Osaka, Japan, in 1985. In 1985, he joined Hokuriku Nippon Electric Software. He became a professor at Kanazawa Institute of Technology, in 2014. His research interests include Embedded System, Software development process and Software

quality management.

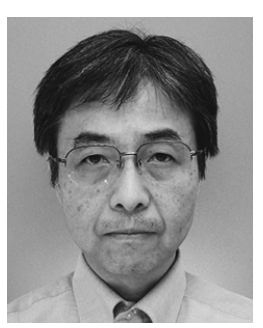

Noriyuki Abe is an associate professor of the Department of Information and Computer Science at Kanazawa Institute of Technology. He received his master degree from Kanazawa Institute of Technology in 1986. He then joined Hitachi. He received his Ph.D. in engineering from Kanazawa Institute of Technology in 1991. He became an assistant professor at Kanazawa Institute of Technology in 1991. Currently, his research interests are autonomous distributed systems and SNS data analysis. He is a member of IPSJ, IEICE, and DBSJ. 